\title{
Mechanism based on pipe cleaning apparatus of high-pressure water jet
}

\author{
Sicheng Nian ${ }^{1, a^{*}}$, Hanxu Sun ${ }^{2, b}$, Yong Cheng ${ }^{3, c}$ \\ ${ }^{1}$ Room 202, Hong Tong Building, Beijing University of Posts and Telecommunications, \\ No. 10 Xi Tu Cheng Road, Beijing, 100876, China. \\ ${ }^{2}$ Room 105, Automation School, Beijing University of Posts and Telecommunications, \\ No. 10 Xi Tu Cheng Road, Beijing, 100876, China. \\ ${ }^{3}$ Room 202, Hong Tong Building, Beijing University of Posts and Telecommunications, \\ No. 10 Xi Tu Cheng Road, Beijing, 100876, China. \\ annssccvery@sina.com, bhx_sun@bupt.edu.cn, ${ }^{\mathrm{c}}$ chengyong@bupt.edu.cn
}

Keywords: High pressure rotating nozzle head, pipeline clean-up program, transmission pipes, adaptive spring system, cams.

\begin{abstract}
Industrial pipes conveying fluid in the long process will produce dirt, which affects the efficiency of transmission. This paper presents a design applicable to pipeline clean-up program. The use of high pressure rotating nozzle head is designed to use high-pressure water jet to clean up the striking force pipeline. This paper also proposes a clamping force relies on two cams to constrain pipes. The coordination with the two cams control the speed and mode of high-pressure pipes to to apply to different pipe scale. And the design of adaptive spring system accommodates different diameter high-pressure transmission pipes. Experimental results demonstrate the effectiveness of pipeline cleaning device based on high pressure water jet.
\end{abstract}

\section{Introduction}

Pipeline in the chemical business has been very widely used, mainly used to transport chemical raw materials and chemical liquid ${ }^{[1,2]}$. The long-time delivery will lead to the chemical liquid remaining in the pipeline, which greatly reduces the effective portion of the pipeline ${ }^{[3]}$, seriously affects the transmission efficiency and increases costs, so that the pipelines need to clean in times ${ }^{[4]}$.

According to the current domestic and international existing high-pressure water jet control scheme, this paper proposes a high-pressure water jet pipe cleaning solution for factories. The use of high pressure rotating nozzle head is designed to use high-pressure water jet to clean up the striking force pipeline ${ }^{[5]}$. The coordination with the two cams control the speed and mode of high-pressure pipes to apply to different pipe scale. And the design of adaptive spring system accommodates different diameter high-pressure transmission pipes. The device is designed to solve the inconvenience to clean up the vertical pipe, and the problems of horizontal pipes' incomplete clean.

\section{Overall high-pressure injection system design and demonstration.}

According to the equation $F=0.745 q \sqrt{p}{ }^{[6]}$ about water jet ( $q$ is the flow per minute through the water, unit L / min, $\mathrm{p}$ is the pressure of the water jet, unit Mpa). The design specifications required of climbing height lead to calculate the pressure and flow pressure, and then adjust the high-pressure pipe nozzle spray angle to achieve the best cleaning effect. Using high-pressure rotating nozzle head, followed by a row of nozzle orifices with a set angle trunk, high-pressure hose connected to the tail through thread. Legs and high pressure nozzle body joints using a torsion spring. The upper legs fit with a large number of small roller. When facing the diameter transformation, torsion springs compress, so that the legs can be shorter to adjust the diameter changing pipeline. The model of high-pressure rotating nozzle head shows in figure 1. 


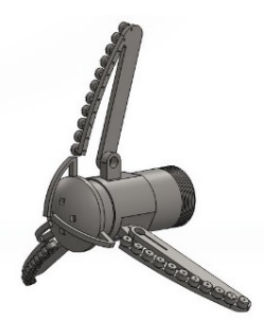

Fig.1 High-pressure rotating nozzle head model

Whether the high pressure hose can go through the variable diameter pipeline, the key point is the choice of torsion stiffness ${ }^{[7]}$. Figure 2 shows some parameters of a torsion spring in large diameter pipe to a small diameter pipe transition state of motion. The analysis of Adams in which the transition from the high-pressure nozzle is designed to process large diameter pipes of small diameter, the role of time and the tail thrust related to the relatively short duration of action. So you can draw a conclusion that the designs of high pressure nozzle thrust can go through varying-diameter pipelines.

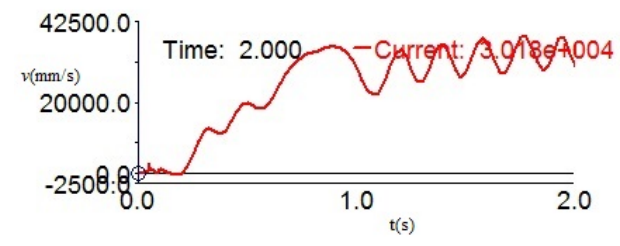

(a) Torsion spring $\mathrm{x}$ direction velocity

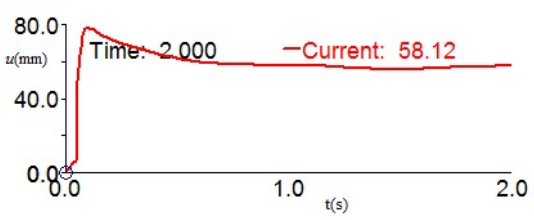

(b) Torsion spring deformation

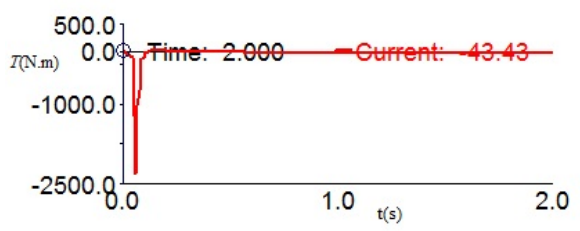

(c) Torsion spring torque diagram

Fig.2 Torsion spring in the variable diameter pipeline motion state diagram

To achieve climbing height, high-pressure rotating nozzle head needs to overcome its own gravity and hose gravity. According to $F=0.745 q \sqrt{p}$, get a group of theoretical value of the pressure and flow rate, the relationship shown in Figure 3. And then according to the theoretical value for the selected motor, and finally a combination of a high pressure injection control system is completed.

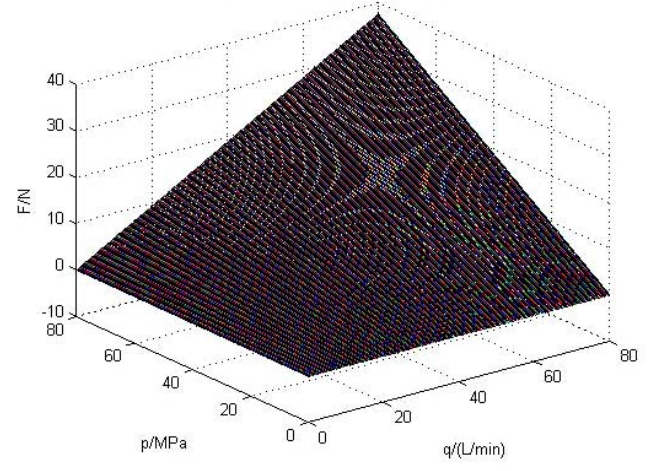

Fig.3 Water jets striking force, pressure and flow diagrams

\section{Design and implementation of high-pressure hose's speed-control mechanism}

Thread screw goes through the hole between the frame and the support plate on the threaded connection. Since the box is fixed, when knobbing screw thread, the thread-support plate will move along the direction of the screw. There are two limit stops on the front end of the movement plate, thereby pressing the spring and the movement plate. The roller mounting shaft passing through the 
movement plate is connected with the passive support rollers, so that the clamping force between motor cam and passive cam can be adjusted. Figure 4 shows the principle diagram of the apparatus used to control the speed.

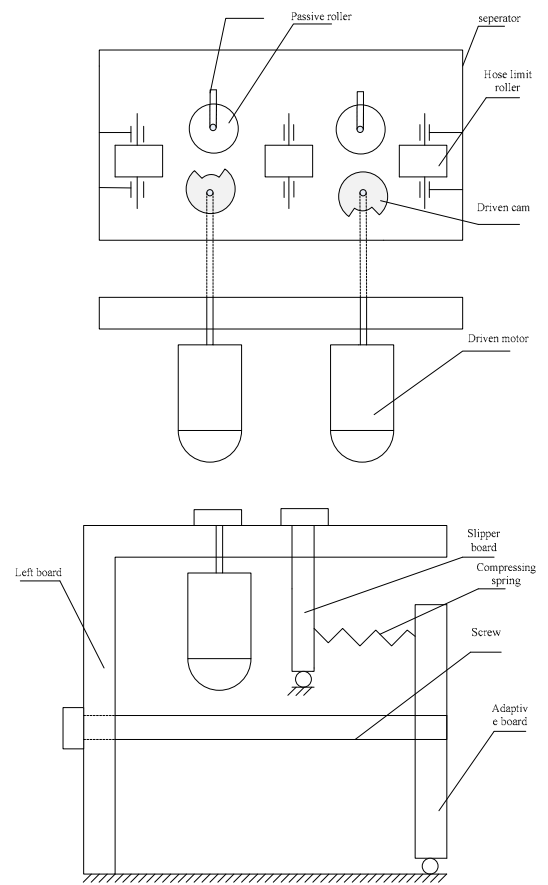

Fig.4 The principle diagram of the apparatus used to control the speed

Combined requirements of the subject, establish a simplified model of stress analysis of the control apparatus, as Figure 5 shows.

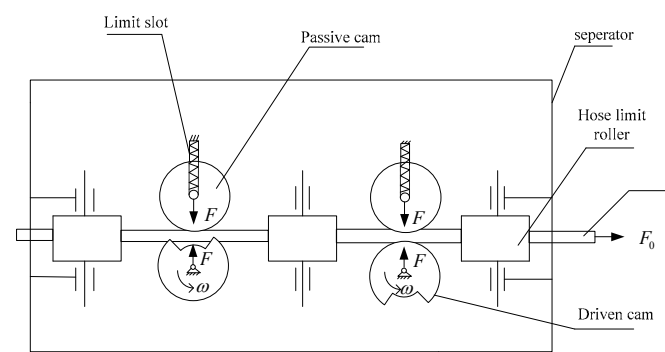

Fig.5 Simplified stress analysis model

The friction produced by motor cam and the driven roller cam of pressurized water pipes must be equal or greater than $F_{0}=150 \mathrm{~N}$, expressed as the equation (1):

$$
\mu F_{\max } \geq F_{0}
$$

The extrusion friction relies on cam motor rotating into the large diameter portion, squeezing the passive roller. While passive roller connected into the separator's limit slot with spring adaptive mechanism by the roller mounting shaft, therefore the pipe extrusion pressure parameters relates to the motor selection and compression springs.

The friction coefficient between the rubber and leather is $0.45^{[8]}$. To ensure the squeeze effect, choose their role efficiency $\eta=75 \%$, from equation $(1)_{F_{\max }} F_{0} / \mu \eta=150 /(0.45 * 0.75)=430 \mathrm{~N}$. That is when the passive wheel compressed to the maximum squeeze, pressure must meet $430 \mathrm{~N}$. According to spring installation size limit (in diameter), take pitch diameter $D=20 \mathrm{~mm}$, diameter $d=4 \mathrm{~mm}$, and effective turns $n=10$, from spring elasticity ${ }^{[9]}$.

$$
k=G d / 8 c^{3} n=G d^{4} / 8 D^{3} n
$$

$\mathrm{G}$ is the spring material parameter, $\mathrm{d}$ is the spring diameter, $\mathrm{D}$ is spring pitch diameter, and $\mathrm{n}$ is the number of effective turns of the spring. From equation (2):

$$
k=8 \times 10^{4} \times 4^{4} /\left(8 \times 23^{3} \times 10\right)=32 \mathrm{~N} / \mathrm{mm}
$$


The two springs are connected in parallel, and their moving distance is $0 \sim 10 \mathrm{~mm}$, so the roller pressure in the range is $0 \sim 640 \mathrm{~N}$ from Hooke's law $F=k \Delta x$, so that we can conclude that selected spring parameters are feasible.

We can adjust the pressure of the spring on passive roller by screw adjustment for spring mounting plate, to achieve the preload adjusting the motor between the driving wheel and the passive wheel. The force diagram is shown in Figure 6.

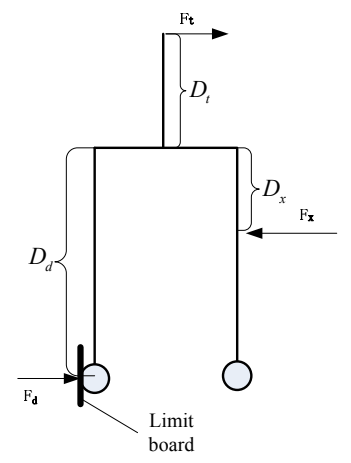

Fig.6 Preload force diagram

The force between spring mounting plate and the moving plate entirely influences by preload adjustment distance $\mathrm{x}, F_{t}$ is the force generated by the restraint slots, $F_{d}$ is the force generated by the limit stops limiting moving plate. Here is equation $(4)^{[10]}$ :

$$
\left\{\begin{array}{l}
F_{x}=F_{d}+F_{t} \\
F_{x} * D_{x}+F_{t} * D_{t}=F_{d} * D_{d}
\end{array}\right.
$$

Introducing preload variable $\mathrm{x}$, from Hooke's law $F_{x}=k \Delta x$ and equation (4), we can get equation (5):

$$
\left\{\begin{array}{l}
F_{t}=F_{x}\left(D_{d}-D_{x}\right) /\left(D_{t}+D_{d}\right) \\
F_{d}=F_{x}\left(D_{d}+D_{x}\right) /\left(D_{t}+D_{d}\right)
\end{array}\right.
$$

$F_{t}$ is passive roller preload, when the drive cam rollers squeeze the water hose and passive cam, preload must be overcome, so that the two pairs of water pressure adjustment can be worked out. When the diameter $\mathrm{x}$ is not the same, the required preload is not the same $F_{t}=\frac{F_{0}-\mu k\left(x-d_{0}\right)}{\mu}$, Diameter relationship between $\mathrm{x}$ and preload is shown in Figure 7.

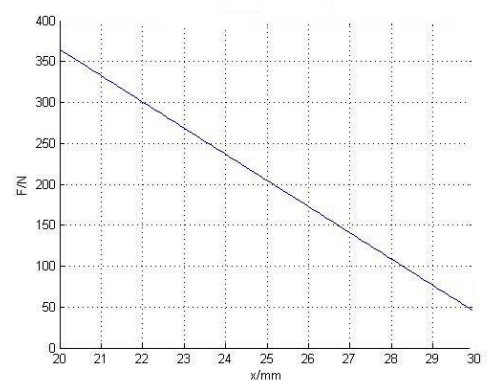

Fig.7 Diameter relationship between $\mathrm{x}$ and preload.

In the water hose transfer process, the motor driven cam drive passive cam to move along restraint slots. There will be a strong roll moment of the moving plate, so an anti-overturning wheel is designed to offset the force and maintain passive restraint slots wheel along a straight line. Force diagram is shown in Figure 8. 


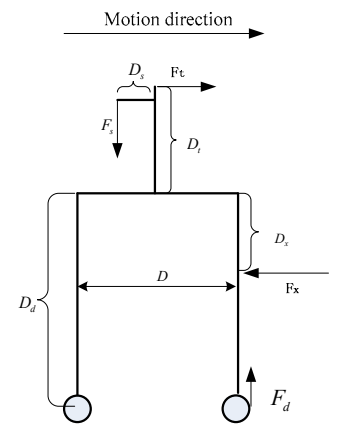

Fig.8 Operation force diagram

Equation 6 is the critical balance equation of overturning the entire organization:

$$
\left\{\begin{array}{l}
F_{s}-F_{d}=0 \\
F_{t}-F_{x}=0 \\
F_{s} * D_{s}+\frac{F_{d}}{2}=F_{x} *\left(D_{x}+D_{t}\right) \\
F_{t}=F_{0} / \mu
\end{array}\right.
$$

Solved:

$$
\left\{\begin{array}{l}
F_{t}=F_{x}=F_{0} / \mu \\
F_{s}=F_{d}=\frac{2 F_{x} *\left(D_{x}+D_{t}\right)}{2 D_{s}+D}
\end{array}\right.
$$

As long as anti-overturning critical equation is met, passive roller can go along a predetermined linear trajectory, to complete its intended function. The apparatus can adapt to the pipes' varied diameter. When changes in diameter, Figure 10 shows the relationship between force $F_{s}$ and diameter, indicating the size of the offset force is related only to the initial force, nothing to do with changes in diameter.

Distribution patterns of different types of pipe cleaning is implemented in software. According to human's judgment about pipe scale type. The mechanism uses two sets of driven and passive roller cam cooperating in harmony. Using the controller for the motor rotation control strategy, more kinds of movement patterns can be worked out. Driven motor cam designs $200^{\circ}$ of $\mathrm{V}$-groove, passive wheel is also designed V-shaped groove, which makes it easy to control water hose closely. When driving cam working in the $200^{\circ}$, the driving cam and passive cam hold the hose to control its speed. And on the other side, the hose is free, so that we can have several ways to control the hose.

\section{Experimental system about pipe cleaning robot and verification}

The water hose speed control mechanism after the simulation and high-pressure injection system after processing and assembly, and develop a good circuit control system joint debugging. Here is the Experimental system to collect relevant data, and assess system performance and improve optimization.

(1) Experimental System Components

The experimental system mainly includes: 1. High-pressure rotating nozzle head 、 2. High-pressure water jet generating device、3. high-pressure hose、4. Arm board 5. Buttons control panel $、 6$. Clamping device(work in pairs)、7.Stepper motors and sensors. Auxiliary equipment includes: ladder stand, pipes enclosed clay and ropes. 4、5、6、7 are in the hose speed-control apparatus. Here are the pipe cleaning robot test system diagram shown in figure 9. 


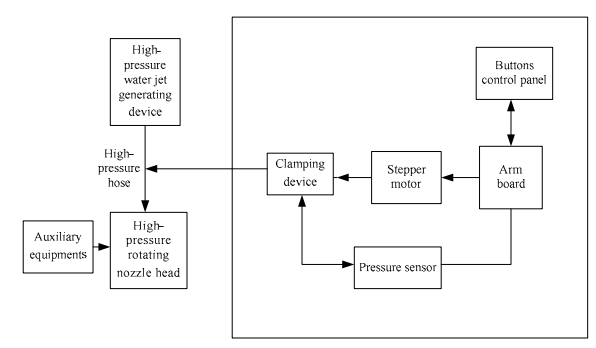

Fig.9 The pipe cleaning robot test system diagram

Strike force produced by high-pressure water jets is used to overcome high-pressure nozzle gravity and gravity to carry water hose. $M=m+\mathrm{K} * x, \mathrm{~K}$ is the hose mass per meter. Figure 10 shows the hose speed control apparatus experimental prototype. Figure 11 shows the high-pressure cleaning equipment pipe cleaning experiment environment.

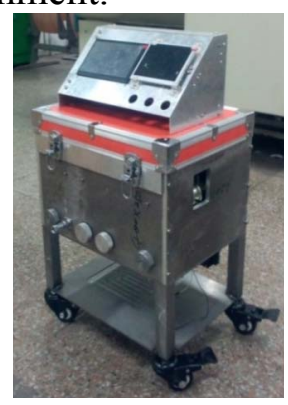

Fig.10 The hose speed control apparatus experimental prototype.
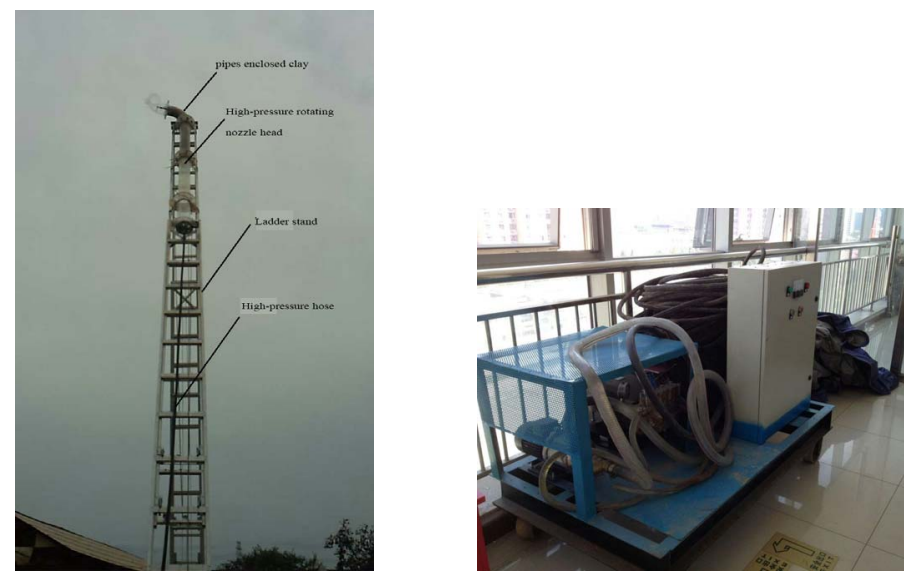

Fig.11 High-pressure cleaning apparatus pipe cleaning experiment environment

Designed according to the above process is completed. The test verifies that the water speed control apparatus can complete three operating modes, display tension curve and transmission distance, so that we can draw a conclusion the design is feasible.

(2) Data processing

The use of screw thread tension sensor installed in both ends of the spring system can adaptively sense extrusion pressure $F_{n}$, and then amplify output signal, finally process by the Arm control panel.

The use of Hall sensor mounted on the rollers can detect wheel rotations times N, whicn can help calculate the distance of the outlet pipe transmission.

(3) Experiment result

To verify the ability to clean up and climbing ability of high-pressure cleaning device, a number of experimental evidences are gotten in the experimental environment. During the cleaning process, a horizontal line through the clean-up mode named "pull and release", enclosed soil simulation pipeline is cleaned very clean. The high pressure nozzle climb to $12 \mathrm{~m}$ ladder to the top and through the bent pipe. Expected results with the experimental results comparison table, as shown in Table 1. 
Table. 1 The results table

\begin{tabular}{|c|c|c|c|c|c|c|}
\hline & $\begin{array}{c}\mathrm{P} \\
(\mathrm{MPa})\end{array}$ & $\begin{array}{c}\mathrm{Q} \\
(\mathrm{L} / \mathrm{min})\end{array}$ & $\mathrm{V}$ & $\begin{array}{c}\mathrm{H} \\
\mathrm{m}\end{array}$ & effect & bent \\
\hline Expect & 80 & 30 & 380 & 15 & Good & Yes \\
\hline result & $0 \sim 80$ & $0 \sim 30$ & 380 & 12 & Good & Yes \\
\hline
\end{tabular}

\section{Summary}

In this paper, the use of high-pressure water jet injection process generated strong striking force to advance the high-pressure nozzle and drive carried high-pressure pipes, and clean pipes. For high-pressure nozzle instability during experiments, design a hose speed control device for speed control: two sets of driven and passive roller cam motor mechanism controller program control is realized by coordination, with a variety of cleaning models to meet different types of pipe cleanup.

Through experiments, mechanism based on pipe cleaning apparatus of high-pressure water jet has a strong climbing ability in the vertical pipes, is capable of carrying high pressure hose climb about $15 \mathrm{~m}$, can complete the pipeline to clean dirt, and is able to adapt to the level of the network pipe. The speed controller can give different ways to control according to pipe condition, complete pipe distribution patterns corresponding data show. This follows mechanism based on pipe cleaning apparatus of high-pressure water jet program is feasible.

\section{References}

[1] Choi H R, Ryew S M. Robotic system with active steering capability for internal inspection of urban gas pipelines,J. Mechatronics. 16(2002) 713-736.

[2] Roh.Se-Gon, Kim.Do-Wan, Lee.Jung-Sub, etal. In-pipe robot based on selective drive mechanism,J. Journal of Control, Automation and Systems. 7(2009)105-112.

[3] Hun-ok Lim, Taku Ohki. Development of Pipe Inspection Robot,C. ICROS-SICE International Joint Conference. (2009)5717-5721.

[4] Mingwei Zhang, Yanheng Zhang,Hanxu Sun. Flexible crawling pipeline robot structure design and the analysis of getting through, J. Development \& Innovation of Machinery \& Electrical Products. 25(2012)6-8.

[5] O.Tatar,C.Cirebea, A.Alutei, etal. The Design of Adaptable Indoor Pipeline Inspection Robots,C. 2010 IEEE International Conference on Automation, Quality and Testing, Robotics. (2010) 325-328.

[6] Wenhui Zhou. Numerical and Simulation Research on the High-Pressure Water Jet Nozzle in Inside and Outside Efflux Flow Field, D. Lanzhou, Lanzhou University of Technology, 2008.

[7] Yanmei Yang, Jinwu Qian,Yanan Zhang. A pipe robot structure and the analysis of curves getting through, J. Design of Machinery. 24(2007)56-58.

[8] Qingkai Li, Dewei Tang, Shengyuan Jiang. Drive characteristics of tri-axial differential in-pipe robot and simulation research,J. Journal of Harbin Engineering University, 33(2012):753-758.

[9] Yinghui Zhang. Elastic body. Mechanical industry press, Beijing, 1980.

[10] Lianggui Pu, Minggang Ji. Design of Machinery,eighth ed. Higher education press, Beijing,2006. 